\title{
Enhancement of the broadband modulation diffraction efficiency of liquid-crystal displays
}

\author{
Jorge Albero, ${ }^{1 *}$ Ignacio Moreno, ${ }^{1}$ and Juan Campos $^{2}$ \\ ${ }^{1}$ Dept. de Ciencia de Materiales, Óptica y Tecnología Electrónica, Universidad Miguel Hernández, o3202 Elche, Spain \\ ${ }^{2}$ Dept. de Física, Universitat Autònoma de Barcelona, 08193 Bellaterra, Spain \\ ${ }^{*}$ Corresponding author: j.albero@umh.es
}

Received September 28, 2011; revised November 16, 2011; accepted November 17, 2011; posted November 18, 2011 (Doc. ID 155360); published December 22, 2011

\begin{abstract}
We report a method to maximize the broadband modulation diffraction efficiency of liquid-crystal spatial light modulators for polychromatic applications requiring a wide range of wavelengths. An optimized encoding pattern based on the minimum Euclidean projection principle is applied in order to increase the diffraction efficiency at large wavelengths that exhibit phase modulation depth lower than $2 \pi$. We demonstrate modulation efficiencies over $80 \%$ on a wavelength range from 454 to $633 \mathrm{~nm}$, which can reach up to $98 \%$ when the range is reduced to $60 \mathrm{~nm}$. Experimental results are shown to confirm the calculations. (c) 2011 Optical Society of America

OCIS codes: $\quad 230.3720,230.6120,050.1970,050.5080,120.5060$.
\end{abstract}

Phase-only diffractive optic elements (DOEs) are often reproduced onto spatial light modulators (SLMs) to profit from their real-time programmable features. Liquidcrystal displays (LCDs) are one of the SLM technologies with a proven ability for DOE displaying. The diffraction efficiency of a DOE is one of its most important properties [1]. When displayed on an SLM, its diffraction efficiency gets reduced due to several effects, such as the limited spatial resolution of the display, its pixel array structure, quantization, nonlinear phase modulation, and coupled amplitude modulation. In the literature, one can find many encoding algorithms to counter these effects [2,3]. Another important issue on modulation efficiency, usually encountered in LCD-SLMs, is a limited phase modulation depth (less than $2 \pi$ ). In this situation, the efficiency can be optimized with a method that consists in addressing the phase by an optimal encoding based on the minimum Euclidean projection [4]. By a so-called saturated mismatched encoding [5,6], an important increase in efficiency is obtained when a display does not reach a phase modulation depth of $2 \pi$.

Phase-only SLMs are also being used in applications with polychromatic light, such as femtosecond laser irradiation [7] and pulse shaping [8], Fourier transform spectroscopy [9], or polychromatic holography [10]. In these applications, chromatic dispersion of the LCD phase modulation can be an issue as well, as reported in the literature [7]. Techniques have been proposed to operate twisted nematic devices in a phase-only modulation mode for a range of wavelengths, although the maximum phase modulation depth is usually very wavelength sensitive $[11,12]$, showing larger values of phase modulation for shorter wavelengths. These differences induce a variation of the modulation diffraction efficiency $\left(\eta_{m}\right)$ versus wavelength. Although this effect can be ignored in some applications and good results can still be obtained [13], a uniform value of $\eta_{m}$ in the operating broadband range is desirable. In fact, devices with reduced dispersion in the phase modulation have been employed in pulse shaping applications []. Moreover, applications such as polychromatic holography could require a high $\eta_{m}$ on a whole wavelength range [13].
In this Letter, we propose a method to maximize and equalize the modulation diffraction efficiency $\eta_{m}$ on a range of wavelengths for which the modulation produced by the LCD is insufficient. Here, we use the abovementioned saturation encoding to enhance $\eta_{m}$ at wavelengths showing reduced phase modulation and, therefore, obtain an encoding pattern that maximizes $\eta_{m}$ on a wavelength range. The proposed encoding will be proven experimentally afterward.

When an LCD addresses a pure-phase function $h(x, y)=\exp [i \varphi(x, y)]$, it incorporates a complex modulation function as $[\underline{5}, \underline{6}]$

$$
m(x, y)=a[\varphi(x, y)] \exp \{i p[\varphi(x, y)]\},
$$

where $a(\varphi)$ and $p(\varphi)$ are the modulus and the phase, respectively, and both are dependent on the addressed phase $\varphi . m(\varphi)$ is the modulation characteristic function of the SLM. Taking into account the $2 \pi$ periodicity of the phase $\varphi$ and assuming that the phase values $\varphi(x, y)$ have a uniform probability distribution in the range $[0,2 \pi]$, $m(\varphi)$ can be expanded in a Fourier series as $[\underline{5}, \underline{6}]$

$$
m(\varphi)=a(\varphi) \exp [i p(\varphi)]=\sum_{\alpha=-\infty}^{\infty} G_{\alpha} \exp (i \alpha \varphi),
$$

where the coefficients of the expansion are calculated as

$$
G_{\alpha}=\frac{1}{2 \pi} \int_{0}^{2 \pi} a(\varphi) \exp [i p(\varphi)] \exp (-i \alpha \varphi) \mathrm{d} \varphi .
$$

If the LCD produces a linear pure-phase-only modulation up to $2 \pi$, all the $G_{\alpha}$ coefficients vanish except $G_{1}=1$. When $m(\varphi)$ shows a nonperfect phase modulation, $G_{1}$ is reduced, and the incoming energy is also diffracted to other orders. $\eta_{m}$ can be defined as the intensity of the $1^{\text {st }}$ diffraction order []], i.e., $\eta_{m}=\left|G_{1}\right|^{2}$.

For simplicity, let us consider a pure-phase modulator, so that the amplitude function $a(\varphi)$ is constant and equal to 1 . Let $p(\varphi)$ range linearly from 0 to a maximum value $\varepsilon=2 \pi(1-c)$, where $c$ is a mismatch parameter in the range $[0,1]$. The ideal working conditions imply that $c=$ 0 at the working wavelength, so the phase modulation 
depth reaches $2 \pi$. Then, from Eq. (3), the intensity of the $1^{\text {st }}$ diffraction order is [2]

$$
\eta_{m}=\left|G_{1}\right|^{2}=\operatorname{sinc}^{2}(c),
$$

where $\operatorname{sinc}(x)=\sin (\pi x) /(\pi x)$. Eq. (4) shows that $\eta_{m}$ is significantly reduced as the parameter $c$ increases.

A solution to increase $\eta_{m}$ when $c>0$ was first proposed in Ref. 5 for monochromatic illumination. It consists in a saturation encoding of the phase function based on the minimum Euclidean projection [4]. The principle lies in following a perfect phase matching up to a maximum modulation depth $\varepsilon$ and approximating each phase on the range $\varphi>\varepsilon$ by the closest available phase, i.e.,

$$
p(\varphi)=\left\{\begin{array}{cc}
\varphi & \varphi<\varepsilon \\
\varepsilon & \varepsilon<\varphi<\varepsilon / 2+\pi . \\
0 & \varphi>\varepsilon / 2+\pi
\end{array}\right.
$$

Eq. (5) leads to an encoding pattern that maximizes $\eta_{m}$, which is then calculated as []ㅡ

$$
\eta_{m}=\left|G_{1}\right|^{2}=\left[\frac{\varepsilon}{2 \pi}-\frac{1}{\pi} \sin \left(\frac{\varepsilon}{2}\right)\right]^{2} .
$$

If we now consider an extended wavelength range, one must introduce the wavelength dependence on the above mathematical expressions. The maximum phase modulation depth $\varepsilon_{\lambda}$ will be wavelength dependent and maximal at the minimum wavelength of the selected range. Let us now assume that $c_{\lambda_{\min }}=0$, so the phase modulation produced by the SLM at the minimum wavelength in the range is maximal, i.e., $\varepsilon_{\lambda_{\min }}=2 \pi$. Our goal is to design a saturated encoding for the displayed DOE that provides the maximum possible $\eta_{m}(\lambda)$ in a selected range of wavelengths. For that purpose, the saturation encoding is applied at a value $\varepsilon_{\mathrm{opt}}$, which is selected as the control parameter to maximize $\eta_{m}(\lambda)$ in the whole range. Adding the wavelength dependence to the three regions defined in Eq. (5), Eq. (6) is now modified to be

$$
\begin{aligned}
\eta_{m}(\lambda)= & {\left[\left(\frac{\varepsilon_{\mathrm{opt}}}{2 \pi}\right) \operatorname{sinc}\left(\frac{\varepsilon_{\lambda}-\varepsilon_{\mathrm{opt}}}{2 \pi}\right)\right.} \\
& \left.+\frac{2}{\pi} \cos \left(\frac{\varepsilon_{\mathrm{opt}}}{4}\right) \sin \left(\frac{\varepsilon_{\lambda}}{2}-\frac{\varepsilon_{\mathrm{opt}}}{4}\right)\right]^{2},
\end{aligned}
$$

where $\varepsilon_{\lambda}$ is the maximum phase depth at a wavelength $\lambda$. When $\varepsilon_{\lambda}=\varepsilon_{\text {opt }}$, Eq. (6) is recovered. We can thus numerically adjust $\varepsilon_{\text {opt }}$ in order to maximize the average $\eta_{m}(\lambda)$ in a range of wavelengths where the behavior of the SLM is known. Note that the saturation encoding at $\varepsilon_{\text {opt }}$ produces an equalization effect on $\eta_{m}(\lambda)$ by reducing the efficiency at wavelengths where $c_{\lambda}$ is close to 0 and increasing the efficiency when it has higher values.

We have experimentally demonstrated this optimization with a parallel-aligned LCD Hammamatsu LCOSSLM X10468 series, which produces phase-only modulation when illuminated with linearly polarized light parallel to the director axis. The SLM has been illuminated with a tunable Ar laser at 454 and $514 \mathrm{~nm}$, as well as with a He-Ne laser operating at $633 \mathrm{~nm}$. The device is adjusted to produce a maximal phase modulation of $2 \pi$ at $\lambda_{\text {min }}=454 \mathrm{~nm}$. Figure $\underline{1(\mathrm{a})}$ shows the function $p(\varphi)$
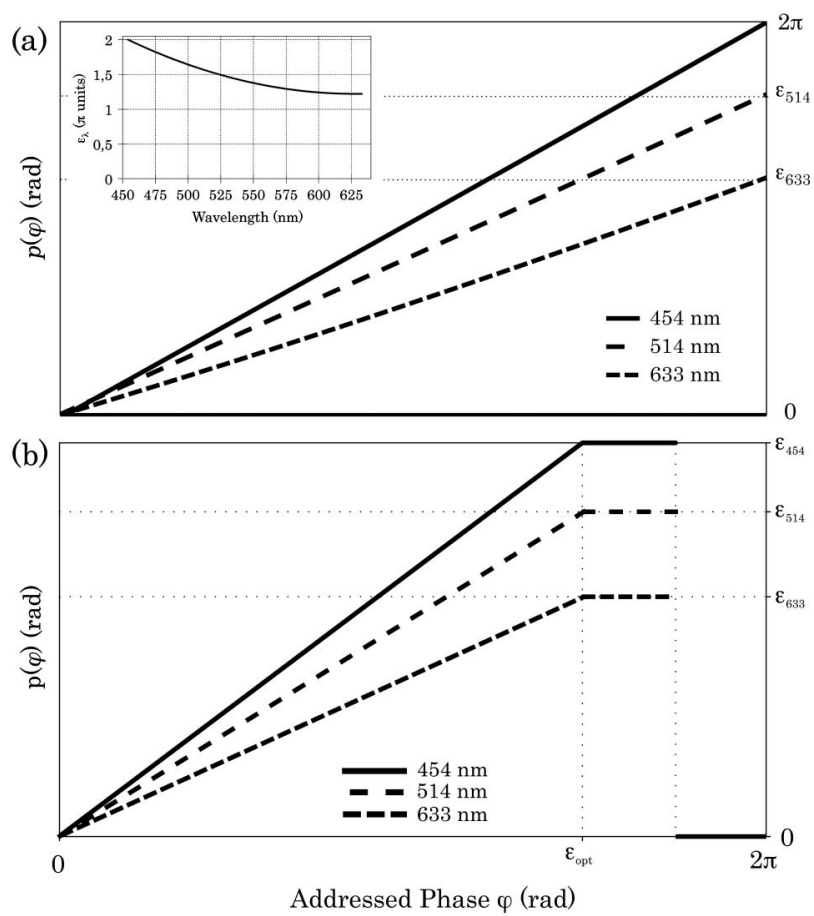

Fig. 1. Characterization of the LCD-SLM used in the experiments. (a) Phase modulation $p(\varphi)$ of each tested wavelength (the inset shows the maximum phase modulation as a function of the wavelength), (b) phase modulation of each wavelength when the broadband optimized encoding is addressed to the LCD-SLM. Both (a) and (b) are represented versus the addressed phase $\varphi$.

for the three selected wavelengths. The maximum phase modulation $\varepsilon_{\lambda}$ of the LCD versus wavelength is shown in the inset of Fig. 1(a), showing the values $\varepsilon_{514}=1,65 \pi$ and
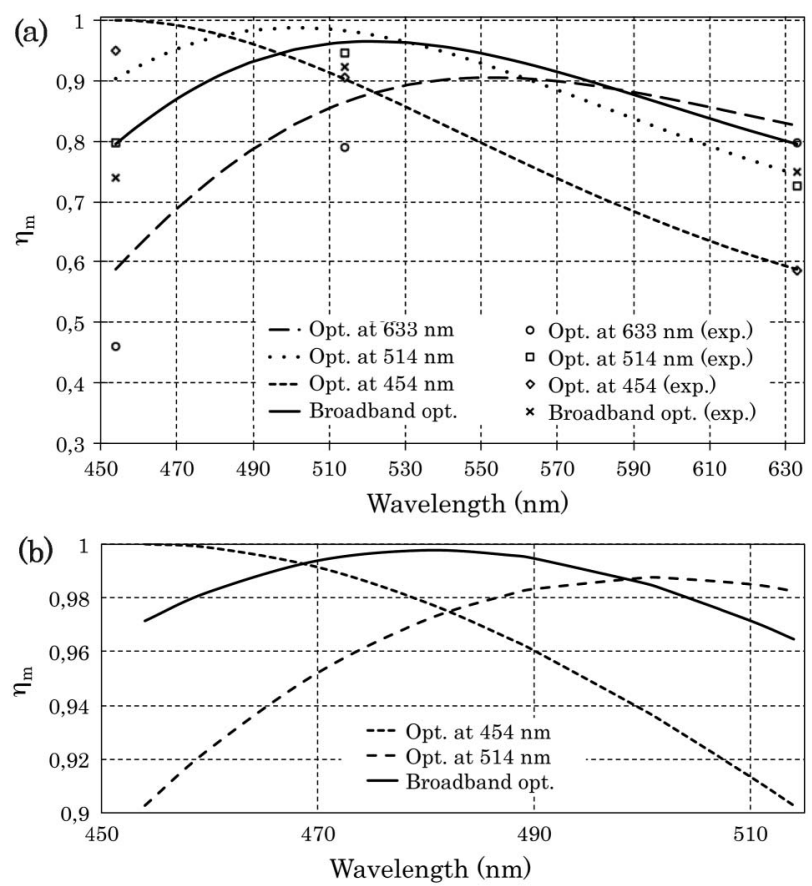

Fig. 2. $\eta_{m}$ versus wavelength (a) in the range 454 to $633 \mathrm{~nm}$ (lines represent calculated values, whereas single points represent experimental results) and (b) in the range 454 to $514 \mathrm{~nm}$. 


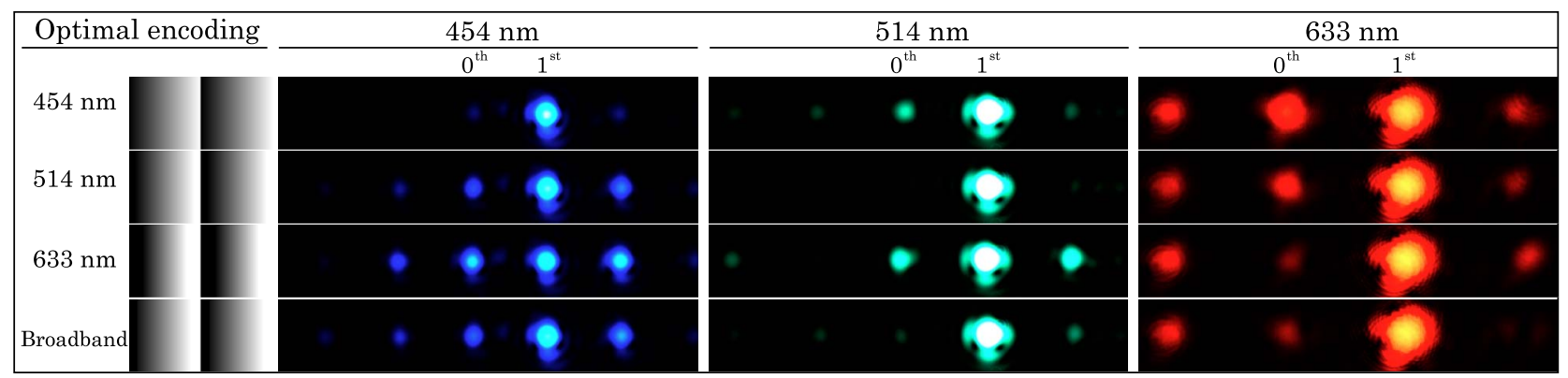

Fig. 3. (Color online) Images of the measured diffracted orders.

$\varepsilon_{633}=1,22 \pi$. The corresponding mismatch parameter $c_{\lambda}$ for the three selected wavelengths are $c_{454}=0$, $c_{514}=0,175$, and $c_{633}=0,391$. Figure $1(\mathrm{~b})$ shows the phase modulation response of these three wavelengths when the broadband optimization encoding is addressed. Here, the phase saturation is selected at the control value $\varphi=\varepsilon_{\text {opt }}$, where each wavelength reaches a maximum value $p(\varphi)=\varepsilon_{\lambda}$.

The curves on Fig. 2(a) represent the normalized diffraction efficiencies of our LCD calculated using Eq. (7) with the $\varepsilon_{\lambda}$ data in the inset of Fig. 1(a). We first calculated $\eta_{m}$ with the optimized encoding for monochromatic illumination at 454,514 , and $633 \mathrm{~nm}$. Note that because $\varepsilon_{454}=2 \pi$, the curve corresponding to $454 \mathrm{~nm}$ matches the response to the linear blaze grating, and therefore the values of $\eta_{m}(\lambda)$ coincide with those given by Eq. (4). Obviously, it produces $100 \%$ efficiency at $\lambda=454 \mathrm{~nm}$, but this gets significantly reduced at the other extreme of the range. On the contrary, when the saturation is produced at $\varepsilon_{633}, \eta_{m}(633)$ is significantly enhanced, but it is very much reduced for the short wavelengths. We then iteratively calculate the value $\varepsilon_{\text {opt }}$ that leads to the maximum average $\left\langle\eta_{m}\right\rangle$ on the complete selected wavelength range, reaching $\varepsilon_{\mathrm{opt}}=1,478 \pi\left(c_{\mathrm{opt}}=0,261\right)$. This broadband optimized encoding predicts values of $\eta_{m}>80 \%$ for almost the complete range, with an average value of $\left\langle\eta_{m}\right\rangle \approx 91 \%$ and a standard deviation $\sigma=6 \%$ (for instance, in the case of the optimization at $454 \mathrm{~nm},\left\langle\eta_{m}\right\rangle \approx$ $82 \%$ with $\sigma=13,5 \%$ ). Since $514 \mathrm{~nm}$ is the wavelength having a phase modulation depth almost in the middle of the range [as shown in Fig. 1(a)], it is clear in Fig. 2(a) how the curve of $\eta_{m}(\lambda)$ obtained with $\varepsilon_{514}$ gives a result near the optimal one obtained with $\varepsilon_{\text {opt }}$.

The experimental measurements [single points on Fig. 2(a)] follow the behavior of the calculations, even though the values are lower due to effects such as losses by the pixel array diffraction effect. Note that the difference is only around $10 \%$ in the worst case. Figure 3 shows the images of the experimental diffracted orders, captured when the different optimal encodings are displayed on the SLM. It is clearly visible that each monochromatic optimized pattern gives the best result at the corresponding design wavelength. We see as well how the broadband optimization gives high $1^{\text {st }}$ order efficiencies for all three wavelengths. Compared to the best monochromatic optimization, the loss of efficiency with the broadband optimization is more visible at $454 \mathrm{~nm}$, in agreement with the calculations.
It must be noted as well that a reduction of the wavelength range increases the overall efficiency. For instance, Fig. 2(b) shows the results when working with a reduced range between 454 and $514 \mathrm{~nm}$. We have now obtained a broadband optimized pattern with $\varepsilon_{\text {opt }}=1,81 \pi$, predicting theoretical efficiencies over $96 \%$ in the whole range and only a standard deviation of $\sigma=1,2 \%$.

In conclusion, we have presented a method to maximize the SLM modulation diffraction efficiency in a wide range of wavelengths. It is achieved by encoding the phase following the saturation scheme derived from the principle of the minimum Euclidean projection. We have validated the method by obtaining efficiencies higher than $80 \%$ in the wide range from 454 to $633 \mathrm{~nm}$.

This work received financial support from Ministerio de Ciencia e Innovación from Spain (ref. FIS200913955-C02-01 and 02) and Conselleria d'Educació i Ciència from Generalitat Valenciana (ACOMP/2011/112). J. Albero acknowledges the financial support from the Ministerio de Educación of Spain through the Programa Nacional de Movilidad de Recursos Humanos del Plan Nacional de I + D + i 2008-2011.

\section{References}

1. D. A. Buralli and G. M. Morris, Appl. Opt. 31, 4389 (1992).

2. M. Kuittinen and H. P. Herzig, Opt. Lett. 20, 2156 (1995).

3. U. Levy, D. Mendlovic, and E. Marom, J. Opt. Soc. Am. A 18, 86 (2001).

4. R. D. Juday, J. Opt. Soc. Am. A 18, 1882 (2001).

5. I. Moreno, J. Campos, C. Gorecki, and M. J. Yzuel, Jpn. J. Appl. Phys. 34, 6423 (1995).

6. I. Moreno, C. Iemmi, A. Márquez, J. Campos, and M. J. Yzuel, Appl. Opt. 43, 6278 (2004).

7. Y. Kuroiwa, N. Takeshima, Y. Narita, S. Tanaka, and K. Hirao, Opt. Express 12, 1908 (2004).

8. J. W. Wilson, P. Schlup, and R. A. Bartels, Opt. Express 15, 8979 (2007).

9. G. Boer, P. Ruffieux, T. Scharf, P. Seitz, and R. Dändliker, Appl. Opt. 43, 2201 (2004).

10. J. Rosen and G. Brooker, Opt. Express 15, 2244 (2007).

11. A. Márquez, C. Iemmi, J. Campos, and M. J. Yzuel, Opt. Lett. 31, 392 (2006).

12. A. Lizana, A. Marquez, I. Moreno, C. Iemmi, J. Campos, and M. J. Yzuel, J. Eur. Opt. Soc. Rapid Publ. 3, 08012 (2008).

13. L. Martínez-León, P. Clemente, E. Tajahuerce, G. MínguezVega, O. Mendoza-Yero, M. Fernández-Alonso, J. Lancis, V. Climent, and P. Andrés, Appl. Phys. Lett. 94, 011104 (2009). 